\title{
LocalLearn: Um modelo U-Learning para auxílio no aprendizado do vocabulário de Inglês
}

\author{
Letícia Garcia da Silva, UNISINOS, leeticia.garcia@gmail.com \\ Rosemary Francisco, UNISINOS, rosemaryf@unisinos.br \\ Jorge Barbosa, UNISINOS, jbarbosa@unisinos.br
}

\begin{abstract}
Resumo. Aprendizes de idiomas geralmente enfrentam problemas de comunicação ao desejarem falar algo e não saberem a palavra ideal. Além disso, os contínuos avanços da tecnologia estão criando novas oportunidades para a aquisição de vocabulário de uma segunda língua por meio da tecnologia. Este artigo apresenta um modelo de aprendizado ubíquo para auxiliar os estudantes de inglês a aprender novos vocabulários. $\mathrm{O}$ estudo avaliou a viabilidade de apoiar o aprendizado de inglês no contexto do usuário. Os resultados indicam uma resposta favorável ao uso do contexto para auxiliar o usuário em sua aprendizagem. Foi alcançada uma aceitação de $78,66 \%$ para a percepção de utilidade e $96 \%$ para a percepção de facilidade de uso.
\end{abstract}

Palavras-chave: Aprendizagem ubíqua, vocabulário de inglês, ciência de contexto.

\section{LocalLearn: An U-Learning model to support English vocabulary learning}

Abstract. Language learners often face communication problems when they need to speak and do not know which word is better. Besides, continuous advances in technology are creating new opportunities for the improvement of second language acquisition purposes through technology. This paper presents a ubiquitous learning model to help English students to learn new vocabulary. The study evaluated the feasibility of supporting English learning in the context of the user. Results indicate a favorable response to the use of the context to assist the user in their learning. An acceptance of $78.66 \%$ for the perception of utility and $96 \%$ for the perception of ease of use was achieved.

Keywords: U-learning, English vocabulary, context science.

\section{Introdução}

A pesquisa realizada por Council (2014) aponta que somente $5,1 \%$ da população brasileira de 16 anos ou mais afirma possuir algum conhecimento do idioma inglês. Um índice baixo, ainda que a língua estrangeira inglesa esteja definida como obrigatória a partir do sexto ano do ensino fundamental, conforme a base nacional comum curricular de ensino do brasileiro (Brasil, 2016). Os estudantes de uma segunda língua geralmente falham em expressar exatamente a mensagem pretendida, devido à falta de conhecimento do vocabulário da língua-alvo. Perceber as falhas no vocabulário facilita o aprendizado incidental de palavras a partir de informações recebidas do ambiente do aprendiz (De Vos et al., 2019).

Atualmente, a aprendizagem de línguas não se limita mais ao contexto tradicional da sala de aula (Hao et al., 2019). O uso crescente da tecnologia mudou a forma como o inglês é aprendido e ensinado (Sundberg; Cardoso, 2019). As tecnologias móveis e ubíquas ampliam as possibilidades de aprendizado de um idioma, permitindo o acesso em múltiplos contextos (Wang et al., 2019). Assim, ao fornecer acesso a um conjunto de recursos e ferramentas, estas tecnologias oferecem vantagens significativas ao V. $17 \mathrm{~N}^{\circ}$ 3, dezembro, 2019 DOI:

RENOTE 
promover o aprendizado de uma segunda língua (Purgina et al., 2019). Além disso, estudos vêm sendo conduzidos para aplicação da computação móvel (Klein et al., 2018) e ubíqua (Cárdenas-Robledo; Peña-Ayala, 2018) na educação.

Este artigo propõe o LocalLearn, um modelo de aprendizagem ubíqua para auxílio ao ensino de vocabulário da língua inglesa. A contribuição científica consiste no uso do contexto do usuário (Dey et al., 2001) e da técnica incidental de aquisição de vocabulário (Xie et al., 2016). Estudos que focam na aquisição de vocabulário assistida por dispositivos móveis vêm se mostrando bastante efetivos. Hao et al. (2019) mencionam que os alunos que usam telefones celulares para aprender o vocabulário em inglês aprendem mais palavras do que aprendendo através de outras mídias.

Além de desenvolver a parte gramatical e vocabulário, os alunos de línguas precisam desenvolver uma disposição para prestar atenção às conexões culturais entre formas, contextos e significados (Purgina et al., 2019). Logo, a vantagem de usar a aprendizagem ubíqua é trazer novas abordagens de aprendizagem, principalmente no que diz respeito as possibilidades de personalização de acordo com o contexto e perfil do aluno (Seixas et al., 2018).

O presente artigo está organizado em 6 seções. A seção 2 apresenta os trabalhos relacionados com o LocalLearn. A seção 3 detalha o método de pesquisa utilizado. $\mathrm{O}$ modelo proposto da solução é apresentado na seção 4 e na seção 5 são apresentados e discutidos os resultados da avaliação. Por fim, na seção 6 são apresentadas as conclusões.

\section{Trabalhos Relacionados}

Sundberg e Cardoso (2019) observaram que é comum se utilizar música em sala de aula para a aprendizagem de uma segunda língua. Os autores utilizaram esta abordagem para construir um modelo de aquisição de vocabulário do Francês. Hao et al. (2019), por sua vez, utilizaram a aprendizagem cognitiva como abordagem pedagógica para a aquisição de vocabulário do Inglês. Os autores desenvolveram um jogo para dispositivos móveis (smartphone Android) com missões que deveriam ser realizadas pelos estudantes. Diferentemente destes modelos, a abordagem pedagógica utilizada pelo LocalLearn é a técnica incidental de aquisição de vocabulário (Xie et al., 2016), visto que estratégias de aquisição de vocabulário incidental auxiliam na motivação do aprendiz.

Purgina et al. (2019) desenvolveram um modelo de aprendizagem da gramática baseado na técnica de aprendizagem de linguagem natural. $O$ modelo permite a configuração da língua a ser aprendida. Esta tarefa deve ser feita pelos professores por meio de uma configuração do conteúdo de aprendizagem em XML. Devido a questões de usabilidade e abordagem pedagógica, o modelo foi desenvolvido apenas para tablets. Os autores também mencionam o uso de elementos de gamificação como suporte para o engajamento dos aprendizes.

Wang et al. (2019) e Liu et al. (2018) desenvolveram modelos de aprendizagem ubíqua com base no uso do contexto dos aprendizes. No entanto, diferente do LocalLearn, os trabalhos utilizaram um contexto específico para a aprendizagem de um conteúdo específico. O conteúdo poderia ser acessado pelo aprendiz por meio de seu smartphone e leitura de um $Q R$ code, disponível no local de aprendizagem. No LocalLearn o contexto de aprendizagem é independente e personalizado, sendo possível realizar uma configuração para o contexto e conteúdo de aprendizagem. O Quadro 1 apresenta uma comparação dos trabalhos relacionados com o modelo proposto, destacando os critérios relevantes considerados para o desenvolvimento do LocalLearn. 
Quadro 1 - Comparativo dos trabalhos relacionados

\begin{tabular}{|c|c|c|c|c|c|c|}
\hline Critérios & $\begin{array}{c}\text { Sundberg e } \\
\text { Cardoso (2019) }\end{array}$ & $\begin{array}{c}\text { Hao et al. } \\
\text { (2019) }\end{array}$ & $\begin{array}{l}\text { Purgina et al. } \\
\text { (2019) }\end{array}$ & $\begin{array}{l}\text { Wang et al. } \\
\text { (2019) }\end{array}$ & $\begin{array}{l}\text { Liu et al. } \\
\text { (2018) }\end{array}$ & LocalLearn \\
\hline $\begin{array}{l}\text { Aquisição de } \\
\text { segunda } \\
\text { língua }\end{array}$ & Sim & Sim & Sim & Sim & Sim & Sim \\
\hline $\begin{array}{l}\text { Foco no } \\
\text { aprendizado }\end{array}$ & Vocabulário & Vocabulário & Gramática & $\begin{array}{l}\text { Leitura e } \\
\text { audição }\end{array}$ & Audição & Vocabulário \\
\hline $\begin{array}{l}\text { Segunda } \\
\text { língua em } \\
\text { foco }\end{array}$ & Francês & Inglês & Configurável & Inglês & Inglês & Inglês \\
\hline $\begin{array}{l}\text { Abordagem } \\
\text { pedagógica }\end{array}$ & $\begin{array}{l}\text { Aprendizagem } \\
\text { por meio de } \\
\text { música }\end{array}$ & $\begin{array}{c}\text { Aprendizagem } \\
\text { cognitiva }\end{array}$ & $\begin{array}{c}\text { Técnica de } \\
\text { aquisição de } \\
\text { gramática de } \\
\text { linguagem } \\
\text { natural } \\
\end{array}$ & $\begin{array}{l}\text { Aprendizagem } \\
\text { de línguas } \\
\text { para fins } \\
\text { específicos }\end{array}$ & $\begin{array}{c}\text { Aprendizagem } \\
\text { colaborativa } \\
\text { de línguas } \\
\text { para fins } \\
\text { específicos } \\
\end{array}$ & $\begin{array}{c}\text { Técnica } \\
\text { incidental de } \\
\text { aquisição de } \\
\text { vocabulário }\end{array}$ \\
\hline $\begin{array}{l}\text { Tipo do } \\
\text { mobile app }\end{array}$ & Web-based app & Native app & $\begin{array}{l}\text { Native app para } \\
\text { tablets }\end{array}$ & Não detalha & Não detalha & Web-based app \\
\hline $\begin{array}{l}\text { Sistemas } \\
\text { operacionais }\end{array}$ & iOS e Android & Android & Android & Não detalha & Não detalha & iOS e Android \\
\hline $\begin{array}{l}\text { Uso do } \\
\text { contexto do } \\
\text { aprendiz } \\
\end{array}$ & Não & Não & Não & $\begin{array}{l}\text { Sim, contexto } \\
\text { específico }\end{array}$ & $\begin{array}{l}\text { Sim, contexto } \\
\text { específico }\end{array}$ & $\begin{array}{l}\text { Sim, contexto } \\
\text { configurável }\end{array}$ \\
\hline $\begin{array}{l}\text { Uso de } \\
\text { Gamificação }\end{array}$ & Não & Sim & Sim & Sim & Não & Não \\
\hline $\begin{array}{l}\text { Método de } \\
\text { pesquisa }\end{array}$ & $\begin{array}{c}\text { Pesquisa } \\
\text { bibliográfica }\end{array}$ & $\begin{array}{c}\text { Quasi- } \\
\text { experimento }\end{array}$ & $\begin{array}{r}\text { Experimento } \\
\text { em sala de aula }\end{array}$ & Experimento & $\begin{array}{c}\text { Quasi- } \\
\text { experimento }\end{array}$ & DSR \\
\hline Público-alvo & $\begin{array}{c}\text { Aprendizes no } \\
\text { geral }\end{array}$ & $\begin{array}{l}\text { Estudantes de } \\
\text { ensino médio }\end{array}$ & $\begin{array}{c}\text { Professores e } \\
\text { estudantes }\end{array}$ & $\begin{array}{c}\text { Aprendizes no } \\
\text { geral }\end{array}$ & $\begin{array}{c}\text { Aprendizes no } \\
\text { geral }\end{array}$ & $\begin{array}{c}\text { Aprendizes no } \\
\text { geral }\end{array}$ \\
\hline $\begin{array}{l}\text { Avaliou a } \\
\text { aplicação }\end{array}$ & Não & Sim & Sim & Sim & Sim & Sim \\
\hline $\begin{array}{l}\text { Utilizou } \\
\text { TAM para } \\
\text { avaliação }\end{array}$ & Não se aplica & Não & Não & Sim & Não & Sim \\
\hline
\end{tabular}

Como apresentado no Quadro 1, o LocalLearn se distingue dos demais trabalhos principalmente no que diz respeito a abordagem pedagógica, por meio do uso da técnica incidental de aquisição de vocabulário; o uso do contexto do aprendiz que pode ser configurável e personalizado, característica importante da aprendizagem ubíqua; além do método de pesquisa adotado, o design science research (DSR).

\section{Metodologia}

O presente estudo adotou o design science research (DSR) (Baskerville et al., 2018) como método de pesquisa. Este método possibilita a construção de uma ampla gama de artefatos sociotécnicos, como o modelo de aprendizagem ubíqua proposto nesta pesquisa. O DSR possibilita resolver problemas de pesquisa de maneira mais eficaz e eficiente, além de fazer contribuições reais e práticas. Desta forma, para a condução do DSR, foram seguidas as etapas propostas por Kuechler e Vaishnavi (2008), conforme apresentado na Figura 1 e detalhado a seguir.

A primeira etapa do DSR consiste na conscientização do problema de pesquisa. Essa etapa foi realizada a partir dos seguintes procedimentos: (1) definição de hipótese do problema de pesquisa de que o contexto do usuário pode auxiliar no seu aprendizado de vocabulário em inglês, (2) revisão da literatura, explorando a abordagem de ensino que a aplicação poderia abranger, como o aprendizado incidental e o inglês para fins específicos, (3) entrevista com duas professoras doutoras que atuam como docentes da língua inglesa em uma Universidade. $\mathrm{O}$ contato com as professoras foi realizado mais de uma vez e possibilitou validar a hipótese e a elaboração de um instrumento (questionário) a ser aplicado em etapa posterior.

A segunda etapa do DSR (sugestão) consiste na aplicação do questionário elaborado na primeira etapa para aprofundar o entendimento do problema junto a potenciais usuários do LocalLearn. Por meio desta coleta foi possível identificar as reais V. $17 \mathrm{~N}^{\circ}$ 3, dezembro, 2019 DOI:

RENOTE 
necessidades dos usuários que deveriam ser endereçadas pelo artefato, o LocalLearn. $\mathrm{O}$ questionário foi operacionalizado por meio do Google Forms e obteve 53 respostas.

Após a definição dos objetivos do artefato, teve início a terceira etapa do DSR, a etapa de desenvolvimento. Esta etapa iniciou com a prototipação da instância do artefato. Os protótipos foram gerados para sistemas operacionais iOS e Android e suas imagens foram anexadas em um questionário para uma primeira avaliação. No questionário foi solicitado que o entrevistado avaliasse as telas quanto a sua percepção de usabilidade, utilidade e entendimento da aplicação. $O$ questionário foi operacionalizado por meio do Google Forms, obtendo um retorno de 7 pessoas.

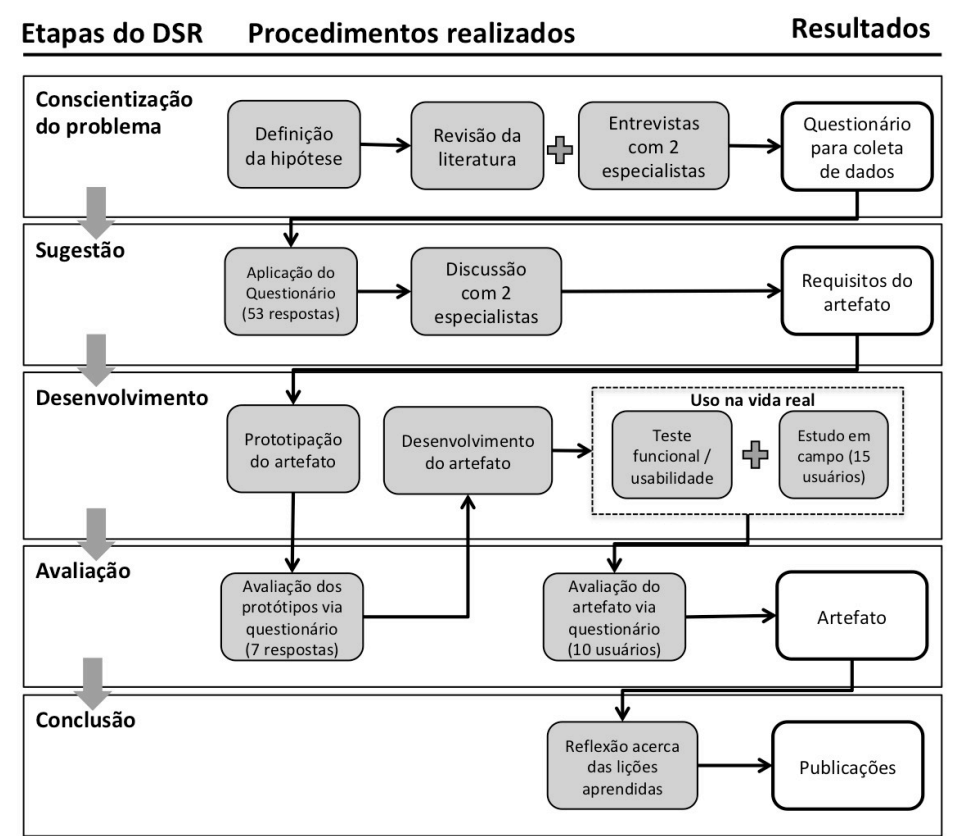

Figura 1. Etapas do DSR

Após a avaliação dos protótipos, iniciou-se o desenvolvimento do LocalLearn e sua instância, uma aplicação móvel. Para avaliar o modelo foram realizados dois procedimentos: primeiramente a aplicação móvel foi instalada e utilizada por 15 usuários. Em seguida foi realizada uma avaliação por meio de um questionário, respondido por 10 usuários. A relação das afirmações do questionário com os aspectos avaliados são mostrados no Quadro 2.

\section{Quadro 2 - Modelo usado para avaliação do LocalLearn}

\begin{tabular}{|l|l|l|}
\hline & Afirmações & Aspecto \\
\hline $\mathbf{1}$ & $\begin{array}{l}\text { O aplicativo LocalLearn me ajudou a aprender e/ou reforçar a compreensão/estudo/aprendizagem do } \\
\text { inglês. }\end{array}$ & PU \\
\hline $\mathbf{2}$ & A repetição de um conteúdo me ajudou a gravar o mesmo mais facilmente. & PU \\
\hline $\mathbf{3}$ & As notificações do aplicativo me incentivaram a utilizar ele com mais frequência. & PU \\
\hline $\mathbf{4}$ & O aplicativo apresentou fácil uso sem a necessidade de ajuda. & PEOU \\
\hline $\mathbf{5}$ & Consegui utilizar de forma satisfatória o aplicativo. & PEOU \\
\hline $\mathbf{6}$ & O aplicativo encontrou corretamente os locais próximos a mim. & C \\
\hline $\mathbf{7}$ & $\begin{array}{l}\text { Ver conteúdos com a localização próxima a mim me incentivou a usar o aplicativo com mais } \\
\text { frequência. }\end{array}$ & C \\
\hline $\mathbf{8}$ & O aplicativo respeitou minhas preferências ao exibir os conteúdos. & C \\
\hline $\mathbf{9}$ & O uso de imagens e exemplos me ajudaram na memorização do conteúdo. & C \\
\hline $\mathbf{1 0}$ & $\begin{array}{l}\text { As informações trazidas dos locais próximos a mim faziam sentido referente ao local em que eram } \\
\text { apresentadas. }\end{array}$ & PUV \\
\hline
\end{tabular}

As afirmações apresentadas no Quadro 2 foram elaboradas com base nos conceitos do modelo de aceitação de tecnologia (Technology Acceptance Model TAM), proposto originalmente por Davis em 1989, e adaptado por Chang, Yan e Tseng V. $17 \mathrm{~N}^{\circ}$ 3, dezembro, 2019 DOI: 
(2012), para a aceitação de tecnologia móvel aplicado na aprendizagem de inglês. Este modelo TAM adaptado considera os seguintes aspectos para o usuário aceitar determinada tecnologia: percepção de utilidade (PU), percepção de facilidade de uso (PEOU), percepção de valor de ubiquidade (PUV) e contexto (C).

\section{Modelo LocalLearn}

Nesta seção são apresentados os detalhes da construção do artefato, o modelo de aprendizagem ubíqua proposto. Inicialmente, é ilustrada a visão geral do modelo, seguida da descrição dos requisitos identificados. Por fim, são apresentados os detalhes da aplicação móvel desenvolvida, a instância do artefato construído, além dos aspectos utilizados para a identificação do contexto do usuário.

\subsection{Visão geral do modelo}

A criação do LocalLearn seguiu os princípios para o desenvolvimento de um modelo CAULL (Context-aware ubiquitous language learning). O modelo tem como intuito suportar a aquisição do vocabulário de Inglês de acordo com o contexto do usuário. Como abordagem pedagógica, o modelo faz uso do aprendizado de vocabulário incidental. Esta aprendizagem refere-se à aquisição de novas palavras em um contexto sem necessariamente o aprendiz ter procurado pela mesma (Xie et al., 2016). Este contexto é observado por meio dos recursos disponíveis nos dispositivos móveis dos usuários.

Os dados do conteúdo de aprendizagem são armazenados em um banco de dados relacional. A troca de dados entre o servidor web e a aplicação é feita por meio de arquivos no formato JSON (JavaScript Object Notation). Para a obtenção dos locais do usuário, através de suas coordenadas geográficas, a aplicação consome dados da API (Application Programming Interface) do Google Places. A Figura 2 ilustra uma visão geral do modelo e a interação entre estes componentes.

O modelo também foi desenvolvido baseado no padrão de projeto MVC (Model View - Controller). O princípio fundamental deste padrão é a divisão da aplicação em três camadas interligadas, de modo a separar a apresentação e interação do usuário com a aplicação, os controles internos da lógica do sistema e o tratamento dos dados (Kilicdagi; Yilmaz, 2014).

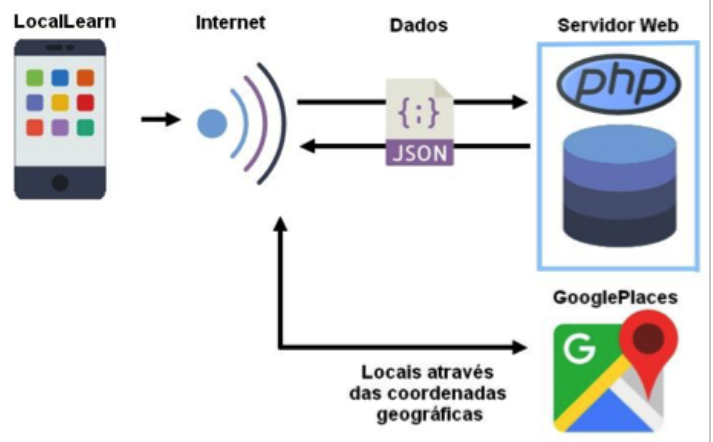

Figura 2 - Visão geral do modelo LocalLearn

\subsection{Requisitos do modelo}

A partir dos resultados da primeira e segunda etapa do DSR (Figura 1), foram identificados os requisitos que o modelo LocalLearn deveria abranger. Os requisitos funcionais estão ilustrados no diagrama de casos de uso mostrado na Figura 3.

Para obter acesso aos conteúdos da aplicação, o usuário deve estar cadastrado e efetuar login (UC03). Em seu primeiro contato com o sistema o usuário realiza o cadastro (UC01) para acesso. Feito esta etapa o usuário é redirecionado para gerenciar V. $17 \mathrm{~N}^{\circ}$ 3, dezembro, 2019 RENOTE DOI: 
suas preferências (UC02), que podem ser alteradas a qualquer momento posteriormente. Assim que as etapas anteriores estiverem concluídas, o sistema obtém a posição geográfica do usuário para enviar ao servidor web a solicitação de conteúdos de acordo com as configurações de localização e perfil de usuário (UC07).

O servidor web por sua vez retorna os conteúdos a serem exibidos (UC04) e armazena o histórico dos conteúdos exibidos por localização (UC06). O usuário tem acesso ao histórico diário resumidamente. Bem como, assim que os conteúdos trazidos pelo servidor web acabarem, o usuário tem a opção de solicitar outros conteúdos (UC05).

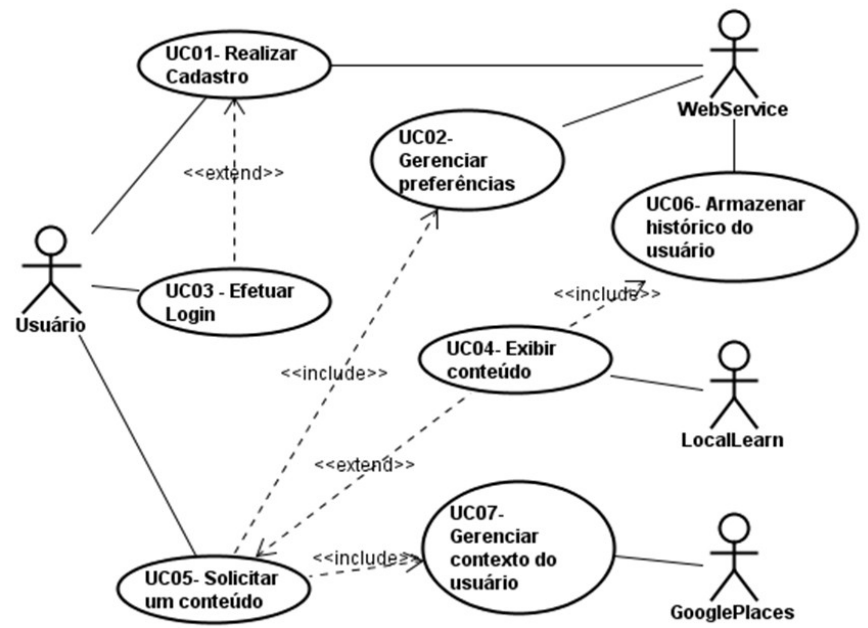

Figura 3 - Requisitos do LocalLearn

\subsection{Aspectos de implementação do modelo}

O modelo foi instanciado, e posteriormente avaliado, por meio de uma aplicação móvel desenvolvida utilizando o framework Ionic. Optou-se por este framework pois o mesmo possibilita a criação de aplicações híbridas (Web-based app), para uso em dispositivos móveis com iOS ou Android. A Figura 4 apresenta três telas da aplicação móvel, correspondentes aos casos de uso UC03, UC04 e UC06, abordados na seção anterior.
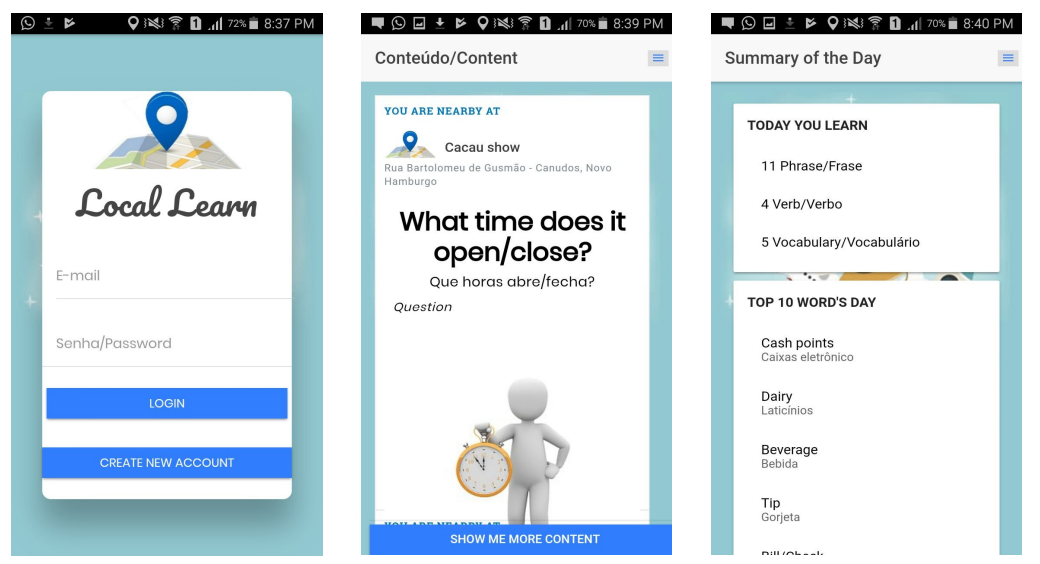

Figura 4 - Telas do aplicativo móvel

Para a gerência do contexto do usuário, a aplicação móvel usa como mecanismo de controle da posição do usuário o plugin Background Geolocation, nativo do Ionic. $\mathrm{O}$ plugin monitora determinado raio, e em caso de deslocamento, muda as variáveis de geolocalização. A posição do usuário é passada para a API Google Places, através da solicitação do método NearBySearch. A solicitação retorna um arquivo JSON com as informações dos locais próximos ao usuário em um raio estipulado por parâmetro. 
Para cada local encontrado nas proximidades, no JSON são retornadas as informações do mesmo. As informações que importam para a aplicação estão em destaque na Figura 5, sendo elas: o nome do local (name), endereço (vicinity) e tipo do lugar (types). É através das informações contidas em Types que a aplicação solicita conteúdos, baseado no contexto, ao servidor web.

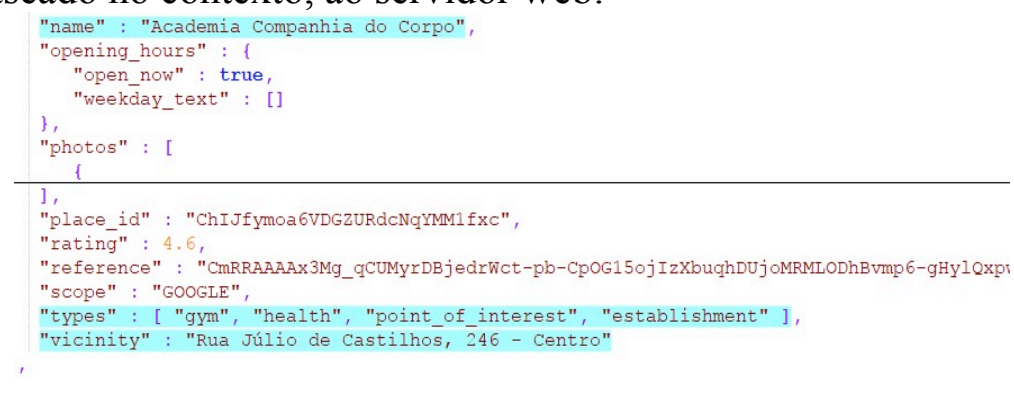

\section{Figura 5 - Retorno NearBySearch de um local próximo ao usuário}

Para efeitos da avaliação, os dados do conteúdo de aprendizagem foram baseados nos 19 tipos de lugares listados na Tabela 1, obtidos através da lista de retorno da API do Google Places. Por meio destes locais o webservice realiza a busca no banco de dados pelos conteúdos de aprendizagem relacionados ao contexto do usuário. Além disso, é avaliada a preferência do usuário por incidência de tipo de conteúdo entre: vocabulário geral, verbos e frases. Os tipos de locais foram escolhidos através de execução da API em testes de coordenadas geográficas, verificando possíveis retornos e pelo critério de diversidade de contextos.

Tabela 1 - Análise dos conteúdos por tipo de local

\begin{tabular}{|c|c|c|c|c|c|c|c|}
\hline \multirow{2}{*}{ Tipo de Local } & \multicolumn{4}{|c|}{ Quantidade de conteúdos por local } & \multicolumn{2}{|c|}{ Histórico } & \multirow{2}{*}{$\begin{array}{c}\text { Média } \\
\text { Aparição } \\
\text { (5)/(4) }\end{array}$} \\
\hline & $\begin{array}{c}\text { Vocabulário } \\
\text { (1) }\end{array}$ & $\begin{array}{c}\text { Verbos } \\
\text { (2) }\end{array}$ & $\begin{array}{c}\text { Frases } \\
\text { (3) }\end{array}$ & $\begin{array}{c}\text { TOTAL } \\
(4)=1+2+3\end{array}$ & $\begin{array}{l}\text { Aparição } \\
\text { p/ usuário } \\
\text { (5) }\end{array}$ & $\begin{array}{c}\text { \% aparição } \\
\Sigma(\mathbf{5}) /(\mathbf{4})\end{array}$ & \\
\hline Academia & 9 & 21 & 17 & 47 & 200 & 4,618 & 4,26 \\
\hline Aeroporto & 11 & 11 & 16 & 38 & 0 & 0,000 & 0,00 \\
\hline Banco & 18 & 10 & 17 & 45 & 429 & 9,905 & 9,53 \\
\hline Bar & 17 & 18 & 28 & 63 & 20 & 0,462 & 0,32 \\
\hline Boate & 11 & 12 & 23 & 46 & 0 & 0,000 & 0,00 \\
\hline Comida & 19 & 16 & 22 & 57 & 541 & 12,491 & 9,49 \\
\hline Escola & 19 & 20 & 30 & 69 & 441 & 10,182 & 6,39 \\
\hline Farmácia & 22 & 7 & 16 & 45 & 37 & 0,854 & 0,82 \\
\hline Hospital & 18 & 12 & 20 & 50 & 231 & 5,334 & 4,62 \\
\hline Imobiliária & 11 & 7 & 10 & 28 & 112 & 2,586 & 4,00 \\
\hline Loja de móveis & 14 & 6 & 20 & 40 & 79 & 1,824 & 1,98 \\
\hline Loja de roupas & 18 & 11 & 21 & 50 & 921 & 21,265 & 18,42 \\
\hline Padaria & 19 & 13 & 16 & 48 & 8 & 0,185 & 0,17 \\
\hline Parada de ônibus & 4 & 15 & 12 & 31 & 0 & 0,000 & 0,00 \\
\hline Restaurante & 24 & 18 & 24 & 66 & 540 & 12,468 & 8,18 \\
\hline Salão de beleza & 10 & 4 & 3 & 17 & 27 & 0,623 & 1,59 \\
\hline Saúde & 13 & 11 & 13 & 37 & 547 & 12,630 & 14,78 \\
\hline Shopping & 25 & 14 & 34 & 73 & 28 & 0,647 & 0,38 \\
\hline Universidade & 23 & 21 & 29 & 73 & 170 & 3,925 & 2,33 \\
\hline
\end{tabular}

\section{Análise e Discussão dos Resultados}

Nesta seção são apresentados os resultados da avaliação do artefato construído, o modelo de aprendizagem ubíqua, instanciado por meio da aplicação móvel desenvolvida. Estes resultados são discutidos com base na literatura. 


\subsection{Avaliação do uso do LocalLearn}

Conforme Tabela 1, foram cadastrados 240 conteúdos distribuídos em 19 tipos de locais, onde um conteúdo poderia aparecer em mais de um local conforme seu contexto. Dentre os tipos de locais, foi constatado que dois deles não tiveram nenhum usuário próximo: aeroporto e parada de ônibus. Portanto, os conteúdos cadastrados para estes locais não foram exibidos. Os locais que tiveram maior incidência para os usuários foram relacionados ao comércio - loja de roupas - e alimentação, restaurante e comida.

\subsection{Avaliação de aceitação do modelo}

A avaliação foi realizada com participantes que fizeram uso de dispositivos Android. Os participantes da segunda etapa do DSR foram convidados a utilizar a aplicação móvel e, posteriormente, responder um questionário de avaliação. Quinze usuários fizeram uso da aplicação e 10 responderam o questionário. O perfil dos participantes foram nove homens e uma mulher com idade entre 16 a 35 anos. As respostas por afirmação são apresentadas na Figura 6.

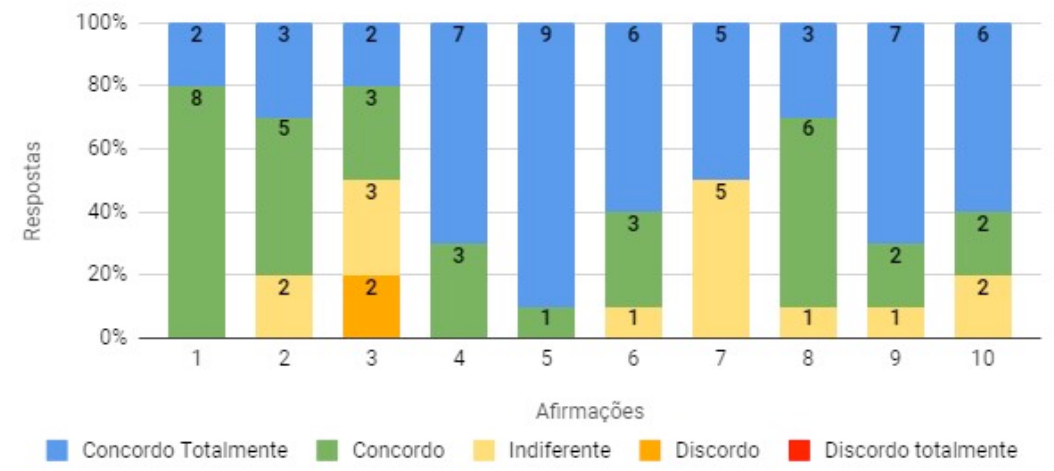

Figura 6 - Resultado da avaliação de aceitação do modelo

Seguindo o mesmo procedimento realizado por Chang, Yan e Tseng (2012), somou-se todos os valores da escala proposta das afirmações correspondentes ao aspecto do TAM e dividiu-se o resultado por: 5 (maior valor da escala) $* \mathrm{~N}^{\mathrm{o}}$ Entrevistados (10) * Quantidade de questões, conforme Tabela 2.

Tabela 2 - Avaliação dos aspectos

\begin{tabular}{|l|l|r|}
\hline Questões & Fórmula & Resultado \\
\hline $1,2,3$ & $\Sigma(1,2,3) \div(5 \times 10 \times 3)$ & 0,7866 \\
\hline 4,5 & $\Sigma(4,5) \div(5 \times 10 \times 2)$ & 0,9600 \\
\hline $6,7,8,9$ & $\Sigma(6,7,8,9) \div(5 \times 10 \times 4)$ & 0,8650 \\
\hline 10 & $\Sigma(10) \div(5 \times 10 \times 1)$ & 0,8800 \\
\hline
\end{tabular}

A percepção de utilidade (correspondente às afirmações 1,2 e 3 ) obteve um grau de concordância de 78,66\%, onde a maior divergência de opinião dos usuários encontra-se na percepção de utilidade para o uso de notificações na aplicação. O aspecto de utilidade foi descrito também pelos usuários como "bastante útil tanto para quem viaja para fora do país tanto para quem vem" (Participante ID02) e "ideia muito interessante para estimular o aprendizado de uma nova língua" (Participante ID09).

$\mathrm{O}$ resultado da percepção de facilidade de uso (correspondente às afirmações 4 e 5) apresentou um índice positivo de aceitação com $96 \%$ de concordância total. Esta percepção também foi evidenciada pelo usuário na questão de livre avaliação, com comentários como: "leve e de fácil compreensão, ou seja, bastante intuitivo" 
(Participante ID02) e "pode facilmente ser utilizado de forma prática no dia a dia" (Participante ID09).

A avaliação do contexto do usuário (correspondente às afirmações 6, 7, 8 e 9) apresentou o resultado de $86,5 \%$ de concordância total. Ao avaliar separadamente as afirmações pode-se observar que para metade dos usuários aprender através da localização foi indiferente para motivar o mesmo em sua frequência de uso.

A utilização de imagens e exemplos foi positivamente aceita pela maioria dos usuários, que também comentaram que "as imagens ajudam muito na memorização" (Participante ID02). Alguns usuários sentiram falta de poder escolher em algum momento o tipo de local que desejavam aprender. Outra questão que foi levantada é o uso de som para a pronúncia. Este ponto vai de encontro com o trabalho de Sundberg e Cardoso (2019) que propõe o uso da música para a aquisição de vocabulário.

A respeito da motivação, um usuário sugeriu utilizar gamificação e interação com redes sociais para incentivar um uso maior da aplicação. Estas sugestões vão de encontro com os achados nos trabalhos de Hao et al. (2019) e Liu et al. (2018) e podem ser implementadas em trabalhos futuros.

\section{Conclusão}

Este estudo apresentou uma resposta favorável ao uso da aprendizagem ubíqua para o auxílio no aprendizado de uma segunda língua. De acordo com o modelo desenvolvido, possibilitou-se aos aprendizes a exposição a novos vocabulários de inglês de forma personalizada, com base no seu contexto. Entre os critérios utilizados para a avaliação do artefato construído, obteve-se aceitação de $78,66 \%$ para a percepção de utilidade, $96 \%$ para a percepção de facilidade de uso, $86 \%$ na avaliação do contexto do usuário e, por último, $88 \%$ de concordância a respeito da ubiquidade. Estes resultados indicam uma receptividade positiva para o LocalLearn, o modelo de aprendizagem ubíqua desenvolvido para auxiliar na aquisição do vocabulário da língua inglesa.

Embora a avaliação do modelo tenha apresentado índices favoráveis, o fato de avaliar com poucos participantes impede generalizações. Sugere-se como trabalhos futuros, a avaliação do LocalLearn com mais aprendizes. Este estudo também demonstra que além da área de ensino de idiomas, a aprendizagem ubíqua é uma oportunidade para futuras pesquisas em relação as diferentes áreas de ensino que podem utilizar a ubiquidade como ferramenta de auxílio e aprendizagem personalizada de acordo com o contexto e perfil do aprendiz.

\section{Referências Bibliográficas}

BASKERVILLE, R. et al. Design Science Research Contributions: Finding a Balance between Artifact and Theory. Journal of the Association for Information Systems, v. 19, n. 5, p. 358-376, maio 2018.

BRASIL. Base Nacional Comum Curricular. Brasília: [s.n.]. Disponível em: $<\mathrm{http}$ //basenacionalcomum.mec.gov.br/images/BNCC_EI_EF_110518_versaofinal_sit e.pdf>.

CÁRDENAS-ROBLEDO, L. A.; PEÑA-AYALA, A. Ubiquitous learning: A systematic review. Telematics and Informatics, v. 35, n. 5, p. 1097-1132, 1 ago. 2018. CHANG, C.-C.; YAN, C.-F.; TSENG, J.-S. Perceived convenience in an extended technology acceptance model: Mobile technology and English learning for college students. Australasian Journal of Educational Technology, v. 28, n. 5, p. 809-826, 26 jul. 2012.

COUNCIL, B. Demandas de Aprendizagem de Inglês no Brasil. São Paulo: [s.n.]. Disponível em: 
$<$ https://www.britishcouncil.org.br/sites/default/files/demandas_de_aprendizagempesqu isacompleta.pdf $>$.

DE VOS, J. F.; SCHRIEFERS, H.; LEMHÖFER, K. Noticing vocabulary holes aids incidental second language word learning: An experimental study. Bilingualism:

Language and Cognition, v. 22, n. 3, p. 500-515, 31 maio 2019.

DEY, A. K.; ABOWD, G. D.; SALBER, D. A Conceptual Framework and a Toolkit for Supporting the Rapid Prototyping of Context-Aware Applications. Human-Computer Interaction, v. 16, n. 2-4, p. 97-166, 12 dez. 2001.

HAO, Y. et al. An evaluative study of a mobile application for middle school students struggling with English vocabulary learning. Computers in Human Behavior, v. 95, p. 208-216, 1 jun. 2019.

KILICDAGI, A.; YILMAZ, H. I. Laravel Design Patterns and Best Practices. [s.1.] Packt Publishing, 2014.

KLEIN, A. Z. et al. The Educational Affordances of Mobile Instant Messaging (MIM). International Journal of Distance Education Technologies, v. 16, n. 2, p. 51-64, 5 abr. 2018.

KUECHLER, B.; VAISHNAVI, V. On theory development in design science research: anatomy of a research project. European Journal of Information Systems, v. 17, n. 5, p. 489-504, 19 out. 2008.

LIU, G.-Z.; CHEN, J.-Y.; HWANG, G.-J. Mobile-based collaborative learning in the fitness center: A case study on the development of English listening comprehension with a context-aware application. British Journal of Educational Technology, v. 49, n. 2, p. 305-320, 1 mar. 2018.

PURGINA, M.; MOZGOVOY, M.; BLAKE, J. WordBricks: Mobile Technology and Visual Grammar Formalism for Gamification of Natural Language Grammar Acquisition. Journal of Educational Computing Research, p. 1-34, 26 fev. 2019. SEIXAS, L. DA R. et al. Design de Aplicação Ubíqua de Aprendizagem para oferecer suporte à conexão entre Cidadãos e o Patrimônio Histórico. Renote, v. 16, n. 2, p. 260 269, 2018.

SUNDBERG, R.; CARDOSO, W. Learning French through music: the development of the Bande à Part app. Computer Assisted Language Learning, v. 32, n. 1-2, p. 49-70, 2 jan. 2019.

WANG, H. et al. Context-aware language-learning application in the green technology building: Which group can benefit the most? Journal of Computer Assisted Learning, v. 35, n. 3, p. 359-377, 20 jun. 2019.

XIE, H. et al. Generating Incidental Word-Learning Tasks via Topic-Based and LoadBased Profiles. IEEE Multimedia, v. 23, n. 1, p. 60-70, 1 jan. 2016. 